University of Nebraska - Lincoln

DigitalCommons@University of Nebraska - Lincoln

$5-1-2000$

\title{
Temperature dependence of magnetic hysteresis of $\mathrm{RCo}_{x}: \mathrm{Co}$ nanocomposites ( $\mathrm{R}=\mathrm{Pr}$ and $\mathrm{Sm}$ )
}

\author{
J. Ping Liu \\ University of Nebraska-Lincoln, pliu@uta.edu \\ Ralph Skomski \\ University of Nebraska-Lincoln, rskomski2@unl.edu \\ Yi Liu \\ University of Nebraska-Lincoln, yliu@unl.edu \\ David J. Sellmyer \\ University of Nebraska-Lincoln, dsellmyer@unl.edu
}

Follow this and additional works at: https://digitalcommons.unl.edu/physicssellmyer

Part of the Physics Commons

Liu, J. Ping; Skomski, Ralph; Liu, Yi; and Sellmyer, David J., "Temperature dependence of magnetic hysteresis of $\mathrm{RCo}_{x}$ :Co nanocomposites (R=Pr and Sm)" (2000). David Sellmyer Publications. 62.

https://digitalcommons.unl.edu/physicssellmyer/62

This Article is brought to you for free and open access by the Research Papers in Physics and Astronomy at DigitalCommons@University of Nebraska - Lincoln. It has been accepted for inclusion in David Sellmyer Publications by an authorized administrator of DigitalCommons@University of Nebraska - Lincoln. 


\title{
Temperature dependence of magnetic hysteresis of $\mathrm{RCo}_{x}$ : Co nanocomposites $(R=\operatorname{Pr}$ and $\mathrm{Sm})$
}

\author{
J. P. Liu ${ }^{\mathrm{a}, \mathrm{b})}$ and R. Skomski ${ }^{\mathrm{a})}$ \\ Behlen Laboratory of Physics, University of Nebraska, Lincoln, Nebraska 68588-0111 \\ Y. Liu ${ }^{\text {a) }}$ \\ Department of Mechanical Engineering, University of Nebraska, Lincoln, Nebraska 68588-0656 \\ D. J. Sellmyer ${ }^{\mathrm{a})}$ \\ Behlen Laboratory of Physics, University of Nebraska, Lincoln, Nebraska 68588-0111
}

\begin{abstract}
The temperature dependence of magnetic hysteresis of $\mathrm{RCo}_{x}: \mathrm{Co}(\mathrm{R}=\mathrm{Pr}$ and $\mathrm{Sm})$ nanocomposite films is reported. These films are prepared by $\mathrm{dc}$ and $\mathrm{rf}$ sputtering and subsequent thermal processing. It is found that the squareness of the hysteresis loops deteriorates with decreasing temperature. This is attributed to the enhanced magnetic anisotropy of the hard phases at low temperatures. The analysis of the magnetic reversal process shows that the anisotropy enhancement leads to a transition from cooperated to independent behavior. This analysis agrees well with the experimental results. (C) 2000 American Institute of Physics. [S0021-8979(00)24708-7]
\end{abstract}

\section{INTRODUCTION}

After the discovery of the experimental evidence for intergrain exchange coupling in $\mathrm{Nd}_{2} \mathrm{Fe}_{14} \mathrm{~B}: \mathrm{Fe}_{3} \mathrm{~B}$ composite, ${ }^{1}$ many theoretical and experimental investigations have been carried out on various exchange-coupled nanocomposite materials,${ }^{2-6}$ with the purpose of producing novel high energy-product permanent magnets. We have systematically studied the magnetic hardening and the intergrain exchange coupling at room temperature in several nanocomposite systems. ${ }^{7-13}$ To obtain a better understanding of the intergrain exchange coupling, we measured the temperature dependence of magnetic hysteresis loops of the nanocomposites over a wide temperature range. It has been found that there is a strong temperature dependence of the magnetic hysteresis. We report in this article our low-temperature results for the $\mathrm{PrCo}_{x}$ :Co and $\mathrm{SmCo}_{x}$ :Co nanocomposite systems, and discuss the physics underlying the temperature dependence.

\section{EXPERIMENT}

The $\mathrm{PrCo}_{x}-\mathrm{Co}$ and $\mathrm{SmCo}_{x}-\mathrm{Co}$ nanocomposite films $(x=3.5)$ were first prepared by sputtering the $\mathrm{PrCo}_{x}$ or $\mathrm{SmCo}_{x}$ targets $(x=3.5)$ and Co target onto glass substrates to form $\mathrm{RCo}_{x} / \mathrm{Co}(\mathrm{R}=\mathrm{Pr}$ or $\mathrm{Sm})$ multilayers. The sputtering was performed in a multiple-gun dc- and rf-magnetron sputtering system. The fraction of the soft Co phase in the films was adjusted by changing the thickness of the Co layers in the multilayers. The sputtering parameters in this investigation (including the argon pressure, the power rates of sputtering targets, and the targets-to-substrate distance) were chosen to produce films with maximum coercivity. The asdeposited multilayer films were then subject to heat treatments which were carried out either in a furnace with

\footnotetext{
a) Also at Center for Materials Research and Analysis.

b)Electronic mail: pliu@coes.latech.edu. Present address: Institute for Micromanufacturing, Louisiana Tech University.
}

vacuum $5 \times 10^{-7}$ Torr or in a rapid thermal annealing device. Generally, high coercivity was obtained only after the heat treatment. It is observed that there is no detectable interdiffussion between the magnetically hard $\mathrm{RCo}_{x}$ hexagonal phases and the magnetically soft Co phase during the heat treatment. However, the stoichiometry of the hard phase is not $\mathrm{RCo}_{3.5}$ but $\mathrm{RCo}_{3}$. Details of the sample preparation and characterization can be found in our previous reports. ${ }^{8-11}$

Magnetization loops were measured at different temperatures by a superconducting quantum interference device (SQUID) magnetometer. Very high coercivities (above 45 kOe) and energy products (above 20 MGOe) have been measured from the films at room temperature. ${ }^{11}$ Other characterization and observation of the nanostructures and magnetic properties have been reported elsewhere. ${ }^{8-13}$

\section{RESULTS AND DISCUSSIONS}

Figure 1 shows hysteresis loops at different temperatures for a $\mathrm{PrCo}_{3.5}$ :Co nanocomposite sample with $17 \% \mathrm{Co}$ in volume. $\mathrm{PrCo}_{3.5}$ is the composition of the target. The actual hard phase in the heat treated thin films is $\mathrm{PrCo}_{3}$, as we discussed in our previous work. ${ }^{13}$ This sample has been given a twostep heat treatment. ${ }^{13}$ For the loops measured below room temperature, only the upper half of the loops (demagnetizing curves) are shown. It is clearly seen from the figure that the squareness of the loops becomes increasingly worse with decreasing temperature. The squareness is defined by the ratio of the area closed by the demagnetizing curve and the coordinates in the second quadrant over the area of the rectangle $M_{r} \times H_{c}$. In Fig. 1, the squareness decreases from 0.89 at $300 \mathrm{~K}$ to 0.51 at $5 \mathrm{~K}$. Small squareness is a direct sign of ineffective intergrain exchange coupling in the nanocomposites (excluding other factors which may also have influence on the squareness). A similar phenomenon has been observed by other groups and was interpreted as a decoupling effect caused by enhanced anisotropy at lower temperatures. ${ }^{14,15}$ As shown by Skomski et al. ${ }^{5,12}$ to achieve effective intergrain exchange interaction, the dimension of 


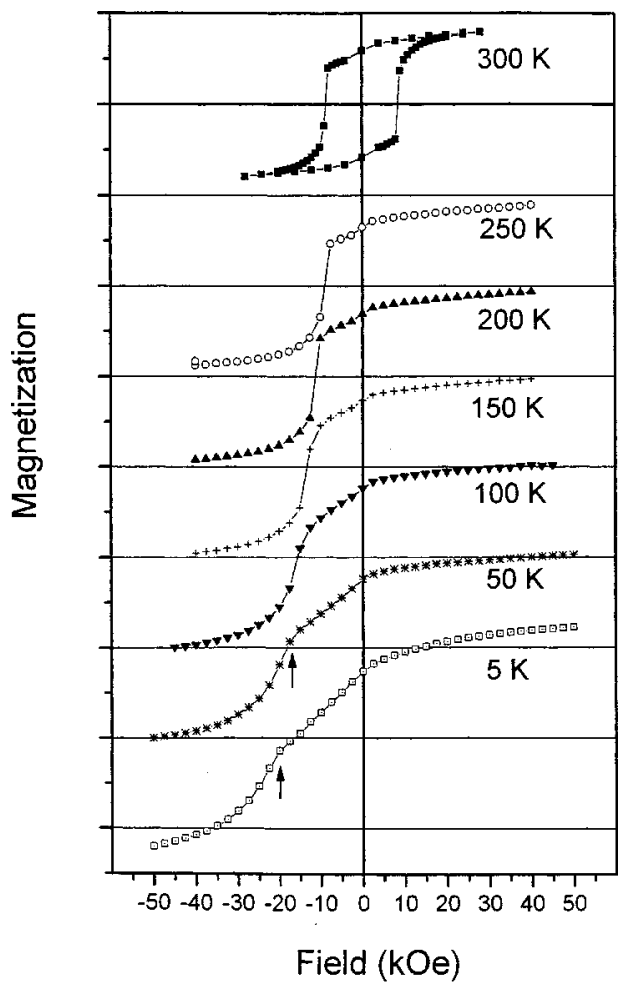

FIG. 1. Magnetic hysteresis loops of the $\mathrm{PrCo}_{x}-\mathrm{Co}$ nanocomposite at different temperatures (below room temperature only the demagnetizing portions are shown). The arrows indicate the switching points.

the soft phase in exchange-coupled composites should not be longer than about twice the domain-wall thickness in the hard phase (this length can be regarded as the relevant exchange length). Usually, at lower temperatures, the domainwall thickness becomes smaller because of the enhanced anisotropy in the hard phases, ${ }^{16}$ which makes the condition for the effective exchange coupling not satisfied anymore, since the grain size does not become smaller with lowered temperature.

This interpretation can be regarded as a qualitative explanation. A more sophisticated description of the magnetic switching process can be established if we analyze the demagnetization curves more carefully. From the demagnetizing curves at temperatures 50 and $5 \mathrm{~K}$ in Fig. 1, we can find transition points in the second or third quadrant (as noted by the arrows). Hysteresis loops with these transitions have low squareness. Similar situation was found in all other nanocomposite samples. Figure 2 shows another example of a $\mathrm{PrCo}_{x}$ :Co nanocomposite sample with the same composition as the sample shown in Fig. 1 but has been given a singlestep heat treatment. It is seen from Fig. 2 that the transition point appears at higher temperature $(100 \mathrm{~K})$ compared with the situation in Fig. 1. This may be caused by the less homogenous soft-phase grain size in this sample (this can be found in Fig. 2 from the shoulders at $H=0$ on the curves at relatively high temperatures). Existence of large soft-phase grains will lead to the violation of the exchange condition at a relatively high temperature. As we discussed in our previous work, ${ }^{13}$ the two-step heat treatment is better in controlling the grain size.

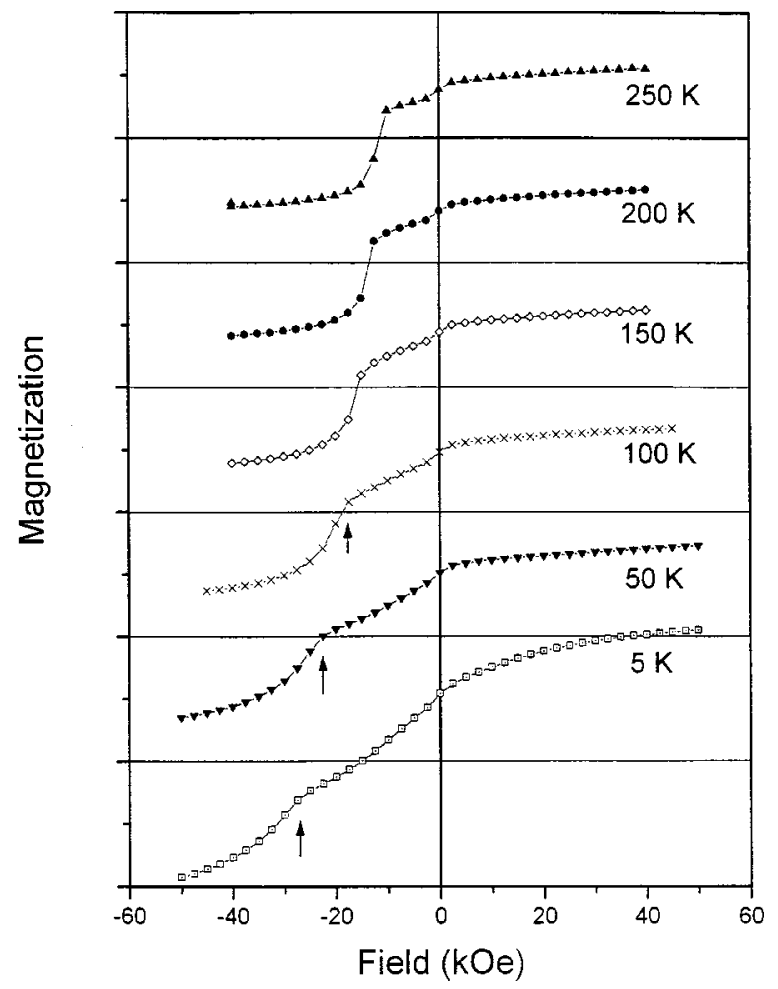

FIG. 2. Magnetic hysteresis loops of the $\mathrm{PrCo}_{x}-\mathrm{Co}$ nanocomposite at different temperatures (only the demagnetizing half branches shown). The arrows indicate the switching points.

These transition points can be attributed to the switching fields for the hard phase in the composites. Demagnetizing curves with such transition points reflect the fact that switching of magnetic moments of the hard phase and soft phase has been separated (not cooperative or simultaneously) at low temperatures. The soft phase has been switched at low fields (near 0 Oe). Higher fields (more negative fields) are necessary to reverse the hard phase because of the enhanced anisotropy. This is why the lower the temperature, the higher the transition fields, as we see from Figs. 1 and 2.

Similar results were found in $\mathrm{SmCo}_{3.5}$ :Co nanocomposite. Figure 3 shows the demagnetizing curves of a Sm system sample with 5\% Co in volume. The shape of the curves and

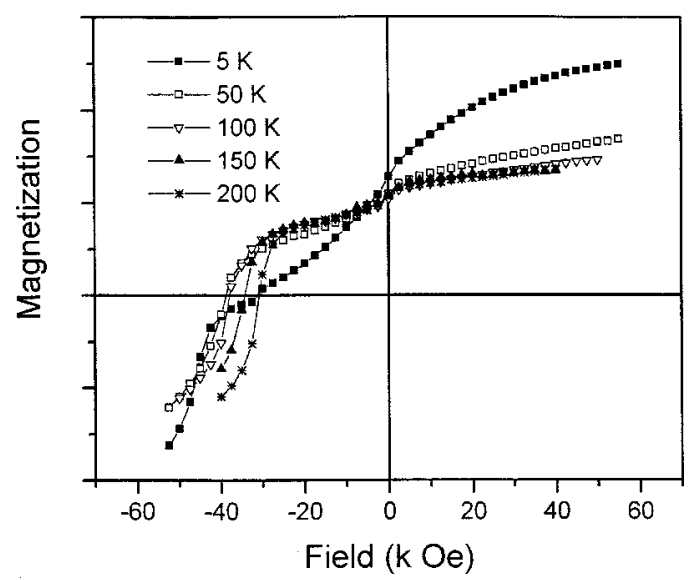

FIG. 3. Hysteresis loops of the $\mathrm{SmCo}_{x}-\mathrm{Co}$ nanocomposites at different temperatures (only the demagnetizing half branches shown). 

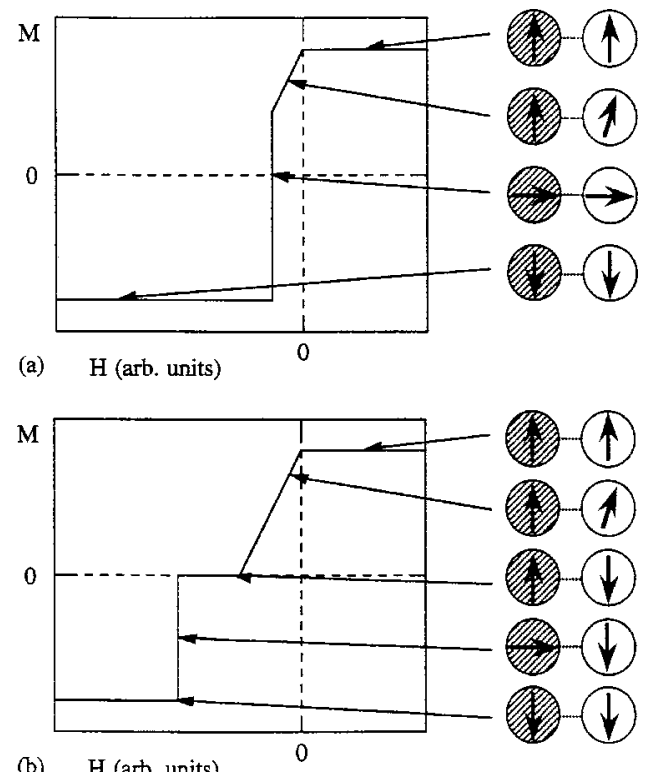

FIG. 4. Modeled hysteresis loop branches: (a) cooperative reversal at high temperatures and (b) independent reversal at low temperatures. For details, see Ref. 19.

their temperature dependence are quite similar as the Pr system samples except that there is a big change between 50 and $5 \mathrm{~K}$. It appears as if there is a significant increase in the anisotropy of the hard phase in this temperature range. No details are available yet.

Our observation of the transition-field dependence on temperature is compatible with susceptibility measurements by O'Sullivan et $a l^{17}$ and the transition points corresponds to the susceptibility maxima in Ref. 17 . The vanishing of the transition point at high temperatures can be interpreted as the onset of cooperative reversal. To elucidate this connection we consider two interacting (exchange-coupled) grains or particles (with exchange stiffness $A$ and particle diameter $L$ ) in an external magnetic field $H$. One grain is hard, characterized by the first anisotropy constant $K_{1}$, whereas the second grain is assumed to be ideally soft. The magnetic energy of this system is

$$
\begin{aligned}
\frac{E}{V}= & \frac{A}{L^{2}}\left(m_{s}-m_{h}\right)^{2}-K_{1}(T) m_{h}^{2}-\mu_{0} M_{0} H m_{s} \\
& -\mu_{0} M_{0} H m_{h} .
\end{aligned}
$$

Here, $V$ is the total volume of the system. The two variables $m_{s}$ and $m_{h}$ describe the magnetization of the soft and hard grains, respectively. Based on this equation, we obtained that at high temperatures, the soft and hard phases will switch simultaneously at a critical field [see Fig. 4(a)], whereas at low temperatures, where $K_{1}$ is high, the reversal will start with the switch of the soft region. Upon further increase of the reverse field, the magnetization of the hard grain switches at a higher field which is directly proportional to $K_{1}$ [see Fig. 4(b)]. This conclusion agrees with our experimental observation.

Cooperative reversal occurs when

$$
K_{1} \leqslant \frac{A}{L^{2}} \text {. }
$$

This means that cooperative reversal occurs at high temperatures, where $K_{1}$ is low. Note that the criterion Eq. (2) for the onset of cooperative behavior is similar to what one finds for cooperative processes in random-anisotropy magnets. ${ }^{18}$ The details of this model will be reported elsewhere. ${ }^{19}$

\section{ACKNOWLEDGMENTS}

This research is supported by AFOSR under Grant No. F49126-98-1-0095. J. P. L and D. J. S are also supported by NSF under Grant No. INT-9812082, and DARPA under Grant No. DAAG 55-98-1-0268.

${ }^{1}$ R. Coehoorn, D. B. de Mooij, and C. de Waard, J. Magn. Magn. Mater. 80, 101 (1989).

${ }^{2}$ P. G. McCormick, W. F. Miao, P. A. I. Smith, J. Ding, and R. Street, J. Appl. Phys. 83, 6256 (1998).

${ }^{3}$ T. Schrefl, J. Fidler, and H. Kronmüller, Phys. Rev. B 49, 6100 (1994).

${ }^{4}$ E. E. Fullerton, J. S. Jiang, C. H. Sowers, J. E. Pearson, and S. D. Bader, Appl. Phys. Lett. 72, 380 (1998).

${ }^{5}$ R. Skomski and J. M. D. Coey, Phys. Rev. B 48, 15812 (1993); Permanent Magnetism (Institute of Physics, Bristol, 1999).

${ }^{6}$ R. F. Sabiryanov and S. S. Jaswal, J. Magn. Magn. Mater. 177-181, 989 (1998).

${ }^{7}$ J. P. Liu, Y. Liu, C. P. Luo, Z. S. Shan, and D. J. Sellmyer, J. Appl. Phys. 81, 5644 (1997).

${ }^{8}$ J. P. Liu, Y. Liu, Z. S. Shan, and D. J. Sellmyer, IEEE Trans. Magn. 33, 3709 (1997).

${ }^{9}$ J. P. Liu, Y. Liu, and D. J. Sellmyer, J. Appl. Phys. 83, 6608 (1998).

${ }^{10}$ J. P. Liu, C. P. Luo, Y. Liu, and D. J. Sellmyer, Appl. Phys. Lett. 72, 483 (1998).

${ }^{11}$ J. P. Liu, Y. Liu, R. Skomski, and D. J. Sellmyer, J. Appl. Phys. 85, 4812 (1999).

${ }^{12}$ R. Skomski, J. P. Liu, M. Meldrim, and D. J. Sellmyer, Proceeding of the Tenth International Symposium on Magnetic Anisotropy and Coercivity in Rare-earth Transition-metal Alloys, edited by L. Schultz and K.-H. Muller, Dresden, September 1998, p. 277.

${ }^{13}$ J. P. Liu, Y. Liu, R. Skomski, and D. J. Sellmyer, IEEE Trans. Magn. 35, 3241 (1999).

${ }^{14}$ D. Goll, M. Seeger, and H. Kronmüller, J. Magn. Magn. Mater. 185, 49 (1998).

${ }^{15}$ G. C. Hadjipanayis, J. Magn. Magn. Mater. 200, 373 (1999)

${ }^{16}$ R. Skomski and M. D. Coey, Permanent Magnetism (Institute of Physics, Bristol, 1999).

${ }^{17}$ J. F. O'Sullivan, P. A. I. Smith, S. David, D. Givord, and J. M. D. Coey, J. Magn. Magn. Mater. 196-197, 182 (1999).

${ }^{18}$ E. Callen, Y.-J. Liu, and J. R. Cullen, Phys. Rev. B 16, 263 (1977).

${ }^{19}$ R. Skomski, J. P. Liu, and D. J. Sellmyer (unpublished.) 